\title{
REEF FISH AND BENTHIC ASSEMBLAGES OF THE TRINDADE AND MARTIN VAZ ISLAND GROUP, SOUTHWESTERN ATLANTIC
}

\author{
Guilherme Henrique Pereira-Filho ${ }^{1,2 *}$, Gilberto Menezes Amado-Filho ${ }^{2}$, Silvia M. P. B. Guimarães ${ }^{3}$, \\ Rodrigo L. Moura ${ }^{4}$, Paulo Y. G. Sumida ${ }^{5}$, Douglas P. Abrantes ${ }^{2}$, Ricardo G. Bahia ${ }^{2}$, Arthur Z. Güth, \\ Renato R. Jorge $e^{3}$ and Ronaldo Bastos Francini Filho ${ }^{6}$
}

${ }^{1}$ Instituto de Pesquisas Jardim Botânico do Rio de Janeiro

(Rua Pacheco Leão, 915, 22460-030 Rio de Janeiro, RJ, Brasil)

${ }^{3}$ Instituto de Botânica

(Av. Miguel Estefano, 3687, 04301-902 São Paulo, SP, Brasil)

${ }^{4}$ Programa Internacional de Conservação Marinha Brasil (Rua das Palmeiras 451, 45900-000 Caravelas, BA, Brasil)

${ }^{5}$ Instituto Oceanográfico da Universidade de São Paulo (Praça do Oceanográfico, 191, 05508-120 São Paulo, SP, Brasil)

${ }^{6}$ Departamento de Engenharia e Meio Ambiente, Universidade Federal da Paraíba (Rua da Mangueira S/N, 58297-000 Rio Tinto, PB, Brasil)

*Corresponding author: rofilho@yahoo.com

\section{A B S TR A C T}

The Trindade and Martin Vaz island group (TMVIG) is located at about 1,120 km off the Brazilian coast. Despite its importance, highlighted by the presence of several endemic fish species, the TMVIG lacks detailed information on the structure of fish and benthic assemblages. Presented here is the first quantitative assessment of reef fish and benthic assemblages of the TMVIG in a depth gradient ranging from 5 to $45 \mathrm{~m}$. Additional qualitative information on reef assemblages between 45 and $100 \mathrm{~m}$ was obtained using advanced gas diving techniques (TRIMIX) and a remotely operated vehicle (ROV). Similarly to other Brazilian oceanic islands, the TMVIG possesses depauperated fish and benthic assemblages, possibly due to its isolation and small size in comparison to the mainland. Depth was the most important factor affecting the structure of fish assemblages, with the density of most fish species declining with depth. Deep reefs $(>45 \mathrm{~m})$ were characterized by the presence of extensive rhodolith beds and rocky reefs sparsely covered with crustose coralline algae, black coral (Cirripathes sp.) and a few massive or plate-like reef corals. Part-time or obligatory planktivorous fishes (e.g. Cephalopholis furcifer and Clepticus brasiliensis) also dominated deep reefs. Similar characteristics were recorded in mesophotic reef ecosystems across the Western Atlantic. Evidence of overfishing (obtained here and in other recent studies), the presence of four endemic and restricted range fish species, as well as the increase in number of new (and still undescribed) endemic taxa, indicates that the adoption of precautionary conservation measures are urgently needed in order to maintain the fragile and unique ecosystems of the TMVIG.

\section{RESUMO}

O conjunto insular de Trindade e Martin Vaz (CITMV) está localizado a aproximadamente $1.120 \mathrm{~km}$ da costa brasileira. Apesar de sua importância, salientada pela presença de diversas espécies endêmicas de peixes, não existem informações detalhadas sobre as assembléias de peixes e bentos do CITMV. É apresentada aqui a primeira caracterização quantitativa das assembléias de peixes e bentos do CITMV em um gradiente de profundidade entre 5 e $45 \mathrm{~m}$. Informações qualitativas adicionais sobre assembléias recifais entre 45 e $100 \mathrm{~m}$ foram obtidas utilizando-se técnicas avançadas de mergulho (TRIMIX) e um veículo de operação remota (VOR). Assim como outras ilhas oceânicas brasileiras, a CITMV possui assembléias depauperadas de peixes e bentos, possivelmente devido ao seu isolamento e pequeno tamanho em comparação ao continente. A profundidade foi o fator que mais afetou a estrutura das assembléias de peixes, com a densidade da maioria das espécies declinando com o aumento da profundidade. Os recifes profundos (> $45 \mathrm{~m}$ ) foram caracterizados pela presença de bancos extensivos de rodolitos e recifes rochosos esparsamente cobertos por algas 
coralináceas incrustantes, corais negros (Cirripathes $\mathrm{sp}$.) e alguns corais massivos e em de forma de placa. Peixes parcialmente ou obrigatoriamente planctívoros (e.g. Cephalopholis furcifer and Clepticus brasiliensis) também dominaram em recifes profundos. Características similares foram registradas para recifes mesofóticos ao longo do Atlântico Ocidental. Evidências de sobrepesca (obtidas aqui e em outros estudos recentes), a presença de quatro espécies de peixes endêmicas e com distribuição restrita, e o aumento no número de espécies novas ainda não descritas, indicam que a adoção de medidas de conservação baseadas no princípio da precaução é urgentemente necessária para garantir a manutenção dos ecossistemas frágeis e únicos do CITMV.

Descriptors: Oceanic islands, Brazil, Reef fish, Benthic assemblages, Habitat characteristics, Mesophotic reefs.

Descritores: Ilhas oceânicas, Brasil, Peixes recifais, Assembléias bentônicas, Características do hábitat, Recifes mesofóticos.

\section{INTRODUCTION}

Oceanic islands typically harbor low species richness and a high proportion of endemic reef fish and benthic species owing to their extreme isolation and relatively restricted shallow water zones (WHITTAKER; FERNANDEZ-PALACIOS, 2007). This high endemism relative to that found in mainland coastal areas makes them priority areas for biodiversity conservation (ALLEN, 2008; FLOETER et al., 2008; KIER et al., 2009). Despite a wealth of qualitative information from Brazilian oceanic islands (e.g. GASPARINI; FLOETER, 2001; FLOETER et al., 2008), there are few quantitative data, either spatial or temporal (but see ROSA; MOURA, 1997; FRANCINI-FILHO et al. 2000; MINTE-VERA et al., 2008), thus precluding the evaluation of their conservation status and the implementation of effective conservation and management measures.

The Vitória-Trindade Chain (VTC) comprises a series of eight seamounts that terminates with a group of emergent islands (hereafter called Trindade and Martin Vaz island group) at its easternmost end, about 1,120 km off the Brazilian coast. The marine biodiversity of Trindade Island is remarkably low; it is considered one of the poorest among tropical islands in the world (FLOETER et al., 2008). Only 129 species of reef fishes (PINHEIRO et al., 2009), 132 species of marine algae (NASSAR, 1994; YONESHIGUE-VALENTIN et al., 2005; VILLAÇA et al., 2006) and 26 species of sponges (MORAES et al., 2006) had previously been recorded on the island.

Logistical issues and the remoteness of Trindade have limited previous studies to qualitative sampling (e.g. NASSAR, 1994; GASPARINI; FLOETER, 2001; PINHEIRO et al., 2009). In addition, most benthic assessments were restricted to the intertidal zone and based on single-day samplings (PEDRINI el al., 1989; NASSAR, 1994), while reef fish assessments used only snorkeling and were thus limited to depths of up to $20 \mathrm{~m}$ (GASPARINI; FLOETER, 2001). The most recent benthic survey, carried out during a large-scale Brazilian EEZ assessment (LAVRADO, 2006), included only a few dredging stations at depths between 30 and $300 \mathrm{~m}$ around the island (YONESHIGUE-VALENTIN et al., 2006). Until now, there has only been one qualitative assessment of reef fish from Martin Vaz Island (PINHEIRO et al., 2009), with no additional data (quantitative or qualitative) on the marine biota of this island. Recent reviews indicate that the marine biota of the Trindade and Martin Vaz island group (TMVIG) is the least known among the Brazilian oceanic islands (ALVES; CASTRO, 2006).

This study is the first quantitative assessment of reef fish and benthic assemblages of the TMVIG for depths ranging between 5 and $45 \mathrm{~m}$. Additional qualitative information on reef fish and benthic assemblages between 45 and $100 \mathrm{~m}$ is provided.

\section{METHODS}

Study area

The areas of the two main islands in the TMVIG $\left(20^{\circ} 30^{\prime} \mathrm{S}, 2^{\circ} 20^{\prime} \mathrm{W}\right.$; Fig. 1$)$ are approximately 10 and $0.5 \mathrm{~km}^{2}$, respectively, with narrow insular shelves, extending about $3 \mathrm{~km}$ from the shore and composed of heterogeneous subvolcanic structures and pyroclastic deposits (MARQUES et al., 1999). The littoral zone is comprised of about $2.5 \mathrm{~km}$ of beaches and $14 \mathrm{~km}$ of narrow $(<1 \mathrm{~km})$ and steep $\left(30-90^{\circ}\right)$ rocky shores. The sublittoral hard bottom is composed of boulders ranging up to several hundred meters in diameter interspersed with patches of sandy/calcareous algae rubble, as well as large uniform rock expanses. The VTC originated from the mantle plume activity along the Vitória-Trindade/Hotspur Fracture Zone which, after erosional events, created eight seamounts with flattened tops at depths of about $50 \mathrm{~m}$ (ALVES et al., 2006). The VTC is mainly under the influence of the Brazilian Current (BC), characterized by warm $\left(>20^{\circ} \mathrm{C} \quad\right.$ SST $)$, relatively saline $(36 \mathrm{psu})$ and oligotrophic waters (SILVEIRA et al., 2000). 


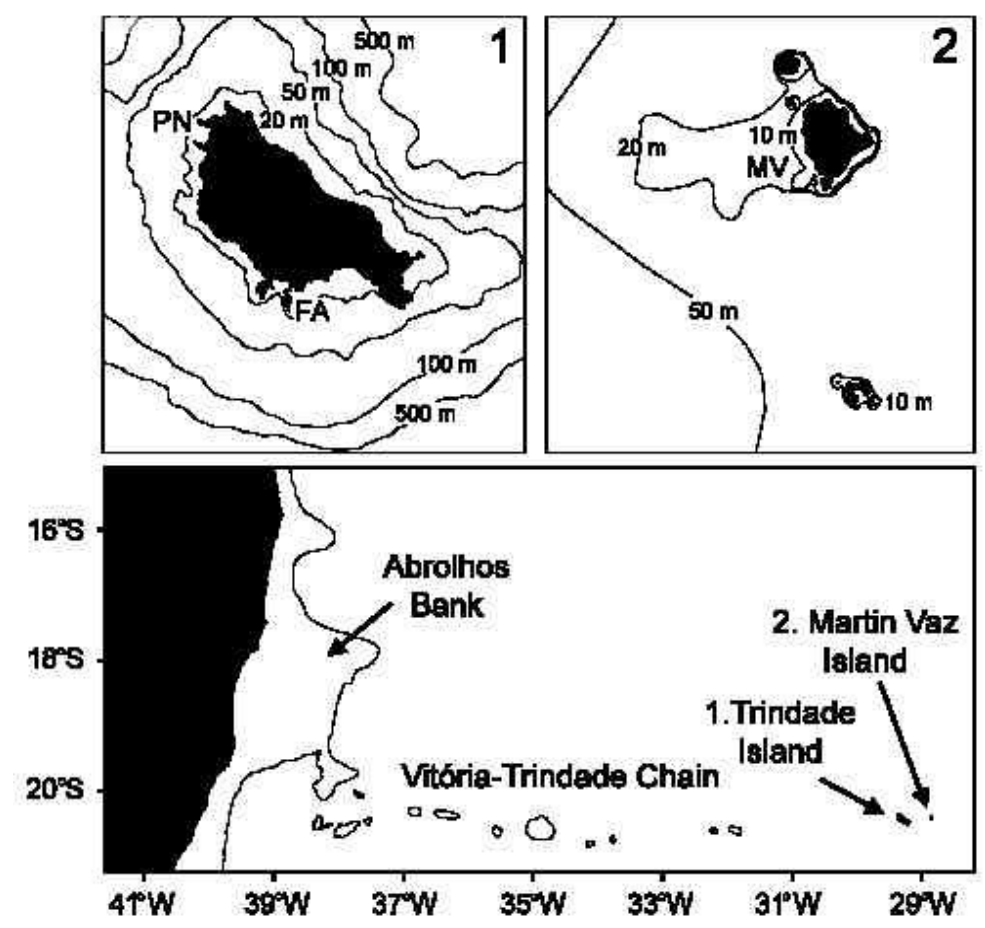

Fig. 1. Map of the eastern Brazilian coast showing the Vitória-Trindade Chain and the Trindade and Martin Vaz island group. Study sites: A) Enseada dos Farilhões (FA), B) Ponta do Noroeste (PN) and C) Martin Vaz Island (MV).

Field Procedures

Surveys were conducted at two sites in Trindade (Ponta Noroeste, PN, and Enseada dos Farilhões, FA) and one site in Martin Vaz (MV) (Fig. 1), between March 16 and 26, 2009. At each site, benthic assemblages were sampled using photoquadrats according to Francini-Filho et al. (2008, 2010). Ten quadrants of $0.7 \mathrm{~m} 2$ were randomly placed along two $50 \mathrm{~m}$ transects parallel to the coast at each site, one at $10 \mathrm{~m}$ and another at $20 \mathrm{~m}$ depth. An additional deeper transect (at about $45 \mathrm{~m}$ depth, which represents the limit of hard bottom) was sampled at FA, along which three quadrants were obtained. Each quadrant was subdivided into fifteen smaller $(0.33 \mathrm{x}$ $0.20 \mathrm{~m}$ ) frames. Thus, each quadrant (sample) was composed of a mosaic of 15 digital images. Benthic percentage cover was estimated with the Coral Point Count with Excel Extension software (CPCe) (KOHLER; GILL, 2006). Fifteen randomly located points were generated per photograph, thus totaling 225 points per quadrant. Organisms immediately below each point were identified to the most precise taxonomic level possible. Quantitative analyses were performed considering the ten following major benthic categories: abiotic (subdivided into bare rock and sand), crustose coralline algae (subdivided into Peyssonelia sp. and others, the latter including mainly Hydrolithon onkodes, Lithophyllum prototypum, Phymatolithon masonianum, Spongites sp.), corals (mainly Siderastrea sp., Montastrea cavernosa and the Brazilian endemic Mussismilia hispida), fleshy macroalgae (cf. STENECK; DETHIER, 2004), octocorals, sponges (subdivided according to color), turf algae (subdivided into: Jania plus Amphiroa plus other small filamentous algae, and the cyanobacteria Lyngbya sp.), and stoloniferous algae (Caulerpa verticilata and C. pusilla). Reference samples of benthic organisms are deposited at both the Jardim Botânico do Rio de Janeiro (JBRJ) and the Instituto de Botânica de São Paulo (IBt-SP).

Reef fish density was assessed using a stationary visual census $(n=10$ per depth strata), following the procedures of MINTE-VERA et al. (2008). Fish counts were carried out at the same sites and depth strata as the benthic sampling with the use of conventional SCUBA. Reference specimens were collected with spears and hand-nets and are deposited 
at the Museu de Zoologia, Universidade de São Paulo (MZUSP).

Qualitative observations on deep reefs (45$100 \mathrm{~m}$ depth) were carried out using advanced mixed gas diving techniques (TRIMIX; $\mathrm{n}=4$ dives) and a remotely operated Seabotix ${ }^{\circledR}$ LBV 150S2 vehicle (ROV) equipped with a color video camera and a pair of scaling lasers $5 \mathrm{~cm}$ apart ( $\mathrm{n}=20$ operations).

\section{Data Analysis}

Calculations were performed for: 1) the 20 most abundant benthic taxa, 2) all major benthic categories, 3) the 20 most abundant reef fish species, and 4) total fish density. Density of large-sized carnivorous fishes (a group particularly susceptible to overfishing; RUSS; ALCALA, 1998) was calculated to allow comparisons with those of protected areas within Brazil, in accordance with fish classification based on data taken from the literature (FRANCINIFILHO; MOURA, 2008; PINHEIRO; GASPARINI, In press) (see Table 1).

Table 1. Reef fish species recorded at Trindade (TR) and Martin Vaz (MV) Islands. Plus sign denotes presence.

\begin{tabular}{|c|c|c|}
\hline FAMILY / SPECIES & TR & MV \\
\hline \multicolumn{3}{|l|}{ Ginglymostomatidae } \\
\hline Ginglymostoma cirratum $^{\mathrm{c}}$ & + & \\
\hline \multicolumn{3}{|l|}{ Carcharhinidae } \\
\hline Carcharhinus perezi $^{\mathrm{c}}$ & & + \\
\hline \multicolumn{3}{|l|}{ Synodontidae } \\
\hline Synodus synodus & + & \\
\hline \multicolumn{3}{|l|}{ Muraenidae } \\
\hline Gymnothorax miliaris & + & \\
\hline Gymnothorax moringa & + & + \\
\hline \multicolumn{3}{|l|}{ Ophichthidae } \\
\hline Myrichthis breviceps & + & + \\
\hline \multicolumn{3}{|l|}{ Holocentridae } \\
\hline Holocentrus adscensionis ${ }^{\mathrm{b}}$ & + & + \\
\hline Sargocentron bullisi ${ }^{\mathrm{b}}$ & + & \\
\hline \multicolumn{3}{|l|}{ Scorpaenidae } \\
\hline Scorpaena plumier & + & \\
\hline \multicolumn{3}{|l|}{ Sphyraenidae } \\
\hline Sphyraena barracuda ${ }^{\mathrm{c}}$ & & + \\
\hline \multicolumn{3}{|l|}{ Serranidae } \\
\hline Cephalopholis fulva ${ }^{\mathrm{b}}$ & + & + \\
\hline Cephalopholis furcifer ${ }^{\mathrm{b}}$ & + & \\
\hline Epinephelus adscensionis $^{\mathrm{c}}$ & + & + \\
\hline Mycteroperca bonaci ${ }^{\mathrm{c}}$ & + & \\
\hline Mycteroperca venenos $a^{\mathrm{b}, \mathrm{c}}$ & + & \\
\hline Rypticus saponaceus & + & \\
\hline \multicolumn{3}{|l|}{ Apogonidae } \\
\hline Apogon americanus & + & \\
\hline Astrapogon stellatus & + & \\
\hline Phaeoptyx pigmentaria & + & \\
\hline \multicolumn{3}{|l|}{ Malacanthidae } \\
\hline Malacanthus plumieri $^{\mathrm{b}}$ & + & \\
\hline \multicolumn{3}{|l|}{ Cirrhitidae } \\
\hline Amblycirrhitus pinos & + & \\
\hline \multicolumn{3}{|l|}{ Carangidae } \\
\hline Carangoides crysos $^{\mathrm{c}}$ & + & \\
\hline Carangoides ruber ${ }^{\mathrm{c}}$ & + & \\
\hline Caranx lugubris $^{\mathrm{BC}}$ & + & + \\
\hline Seriola rivoliana ${ }^{\mathrm{c}}$ & & \\
\hline
\end{tabular}

\begin{tabular}{|c|c|c|}
\hline \multicolumn{3}{|l|}{ Haemulidae } \\
\hline Anisotremus surinamensis & + & \\
\hline \multicolumn{3}{|l|}{ Sparidae } \\
\hline Diplodus argenteus & + & + \\
\hline \multicolumn{3}{|l|}{ Mullidae } \\
\hline Pseudupeneus maculatus & + & \\
\hline Mulloidichthys martinicus $^{\mathrm{b}}$ & + & \\
\hline \multicolumn{3}{|l|}{ Chaetodontidae } \\
\hline Chaetodon striatus & + & \\
\hline Prognathodes brasiliensis ${ }^{\mathrm{b}}$ & + & + \\
\hline \multicolumn{3}{|l|}{ Pomacanthidae } \\
\hline Centropigy aurantonotus & + & \\
\hline Holacanthus tricolor ${ }^{\mathrm{b}}$ & + & \\
\hline \multicolumn{3}{|l|}{ Kyphosidae } \\
\hline Kyphosus sectator & + & + \\
\hline \multicolumn{3}{|l|}{ Pomacentridae } \\
\hline Abudefduf saxatilis & + & + \\
\hline Chromis flavicauda $^{\mathrm{b}}$ & + & \\
\hline Chromis jubauna $a^{\mathrm{a}, \mathrm{b}}$ & + & \\
\hline Chromis multilineata & + & + \\
\hline Microspathodon chrysurus & + & + \\
\hline Stegastes pictus ${ }^{\mathrm{b}}$ & + & + \\
\hline Stegastes fuscus trindadensis & + & + \\
\hline \multicolumn{3}{|l|}{ Labridae } \\
\hline Bodianus pulchellus ${ }^{\mathrm{b}}$ & + & \\
\hline Bodianus rufus & + & \\
\hline Clepticus brasiliensis $^{\mathrm{b}}$ & + & \\
\hline Halichoeres brasiliensis & + & + \\
\hline Halichoeres rubrovirens & + & + \\
\hline Halichoeres poeyi & + & + \\
\hline Thalassoma noronhanum & + & + \\
\hline \multicolumn{3}{|l|}{ Scaridae } \\
\hline Sparisoma amplum & + & \\
\hline Sparisoma axillare & + & \\
\hline \multicolumn{3}{|l|}{ Trypterygiidae } \\
\hline Enneanectes sp. & + & \\
\hline \multicolumn{3}{|l|}{ Labrisomidae } \\
\hline Labrisomus nuchipinnis & + & + \\
\hline Malacoctenus brunoi & + & + \\
\hline \multicolumn{3}{|l|}{ Bleniidae } \\
\hline Entomacrodus sp. & + & \\
\hline Hypleurochilus sp. & + & + \\
\hline Ophioblennius trinitatis & + & + \\
\hline Scartella poiti & + & + \\
\hline \multicolumn{3}{|l|}{ Gobiesocidae } \\
\hline $\operatorname{Arcos} \mathrm{sp}$. & + & \\
\hline \multicolumn{3}{|l|}{ Gobiidae } \\
\hline Coryphopterus sp. & + & \\
\hline Elacatinus pridisi & + & + \\
\hline Acanthuridae & & \\
\hline Acanthurus bahianus & + & + \\
\hline Acanthurus coeruleus & + & + \\
\hline Balistidae & & \\
\hline Balistes vetula & + & + \\
\hline Canthidermis sufflamen $^{\mathrm{b}}$ & + & + \\
\hline Melichthys niger & + & + \\
\hline Monacanthidae & & \\
\hline Aluterus scriptus & + & \\
\hline Cantherhines macrocerus & + & + \\
\hline Cantherhines pullus & + & + \\
\hline Tetraodontidae & & \\
\hline Canthigaster figueiredoi & + & \\
\hline Sphoeroides spengleri & + & + \\
\hline Diodontidae & & \\
\hline Diodon holacanthus & + & \\
\hline Diodon hystrix & + & \\
\hline Ostraciida & & \\
\hline Acanthostracion polygonius ${ }^{\mathrm{a}}$ & + & \\
\hline
\end{tabular}

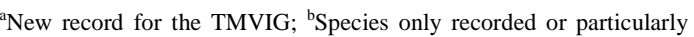
abundant on deep reefs (> $60 \mathrm{~m}$ ); ${ }^{c}$ Large carnivorous fishes targeted by fisheries. 
Two-way analysis of variance (ANOVA) was used to evaluate differences in benthic coverage and fish density between sites and depths. The deeper transect at the FA site was excluded from the ANOVA due to lack of sampling in a similar depth stratum at the other sites. Normality and homoscedasticity were improved by converting percentage values of benthic cover to $\arcsin \sqrt{\mathrm{X}}$ and fish density estimates to $\log$ $(\mathrm{x}+1)$. Post-hoc comparisons were performed using the Tukey test (ZAR, 1999). Linear regression was used to evaluate the relationship between depth and total fish density, with both variables previously converted to $\log (x+1)$. Samples from the deeper strata at the FA site were included in this latter analysis.

Non-metric multidimensional scaling (nMDS) was used to summarize similarities (BrayCurtis) in benthic and fish assemblage structures between sites and depths, and a two-way analysis of similarity (ANOSIM) was performed to evaluate significant differences among sites (CLARKE; WARWICK, 1994). A fourth root transformation was performed prior to the analyses in order to avoid the excessive weight of numerically dominant species (CLARKE; WARWICK, 1994). Canonical correspondence analysis (CCA) (TER BRAAK, 1996) was used to evaluate the influence of habitat characteristics (i.e. benthic coverage and depth) on the structure of fish assemblages.

\section{RESULTS}

\section{Fish Assemblages}

A total of 73 species of fish belonging to 33 families were recorded (Table 1), representing about $67 \%$ of the known reef fish species pool of the TMVIG (GASPARINI; FLOETER, 2001; PINHEIRO et al., 2009). Total fish density varied significantly between sites, with the highest values recorded at the MV and PN sites, respectively. Total fish density declined with depth (linear regression: $F=23.3 ; P<$ $0.001 ; R=-0.54$ ), the same pattern being recorded for all fish species for which significant between-depth strata differences were recorded (see post-hoc comparisons in Table 3). The twenty most abundant fish species are shown in Figure 2. Four species (Cephalopholis fulva, Chromis multilineata, Halichoeres rubrovirens and Stegastes fuscus trindadensis) were more abundant at the MV or at the MV plus PN sites, while three species (Abudefduf saxatilis, Acanthurus bahianus and Malacoctenus brunoi) were more abundant at the PN or at the PN plus FA sites. There were no differences in density of large carnivorous fishes between sites and depth strata, with the overall density value (i.e., of all sites pooled) estimated at $0.03 \pm 0.01$ individuals. $\mathrm{m}^{-2}$. No other significant differences between sites and/or depth strata were recorded (Fig. 2; Table 3). The structure of fish assemblages differed significantly between both sites $(R=0.25 ; P=0.001)$ and depth strata $(R=$ $0.56 ; P=0.001$; Figure $4 \mathrm{a})$, with a stronger difference (i.e., higher $R$ values) recorded for the latter.

Qualitative observations on deep reefs $(>60 \mathrm{~m})$ showed that 16 species were dominant (see Table 1). Part-time or obligatory planktivorous fishes (Cephalopholis furcifer, Mulloidichthys martinicus, Chromis flavicauda, Chromis jubauna and Clepticus brasiliensis) were particularly abundant in deeper areas. Fishes were mostly associated with prominent and complex bottom features (e.g. Malacanthus plumieri nests composed of piles of rhodoliths and rock expanses covered mainly by black corals near the break of the narrow insular shelf; see below).

\section{Benthic Assemblages}

A total of 51 benthic taxa were recorded. The three new records of corals and 11 new records of macroalgae for the TMVIG are worth noting (Table 2). In addition, the crustose coralline algae, Lithophyllum prototypum (Martin Vaz Island, $20^{\circ} 28^{\prime} 05^{\prime}$ 'S,$\quad 28^{\circ} 51^{\prime} 28^{\prime}$ 'W, GM Amado-Filho, 22.III.2009, RB 498282), represents a new record for the entire South Atlantic Ocean.

Sand was the dominant type of benthic cover, followed by Caulerpa verticillata, crustose coraline algae (excluding Peyssonelia sp.), Dictyota mertensii and Canistrocarpus cervicornis. The other common benthic organisms are shown in Figure 3. The benthic cover of two reef corals (Mussismilia hispida and Siderastrea sp.), the stoloniferous algae Caulerpa pusilla and the cyanobacteria Lyngbya sp., was greater at the MV site. Four taxa (Peyssonelia sp., Canistrocarpus cervicornis, green sponges, and Jania plus Amphiroa turf) dominated at the FA site, while two fleshy macroalgal species (Dictyota ciliolata and D. mertensii) were more abundant at the PN site (Fig. 3; Table 4). No other significant differences between sites and/or depth strata were recorded. Similarly to fish assemblages, the structure of benthic assemblages differed significantly between the two sites $(R=0.86$; $P=0.001)$ and depth strata $(R=0.57 ; P=0.001$; Fig. $4 \mathrm{~b}$ ), with a stronger effect of the former, as indicated by the higher $R$ value.

The shallow benthic realm was replaced by a deep reef assemblage at about $45 \mathrm{~m}$, this latter comprised of a mosaic of small sandy patches and rhodolith pavement. From 80 to $90 \mathrm{~m}$ depth, the rhodolith pavement was substituted by a narrow steep rocky reef $\left(\sim 35^{\circ}\right)$, which marks the end of the insular shelf. These deep rocky reefs were sparsely covered with crustose coralline algae, colonies of the black coral Cirripathes sp., as well as the plate-like and massive reef corals Agaricia cf. fragilis, Montastrea cavernosa and Scolymia wellsi (see Table 2). 


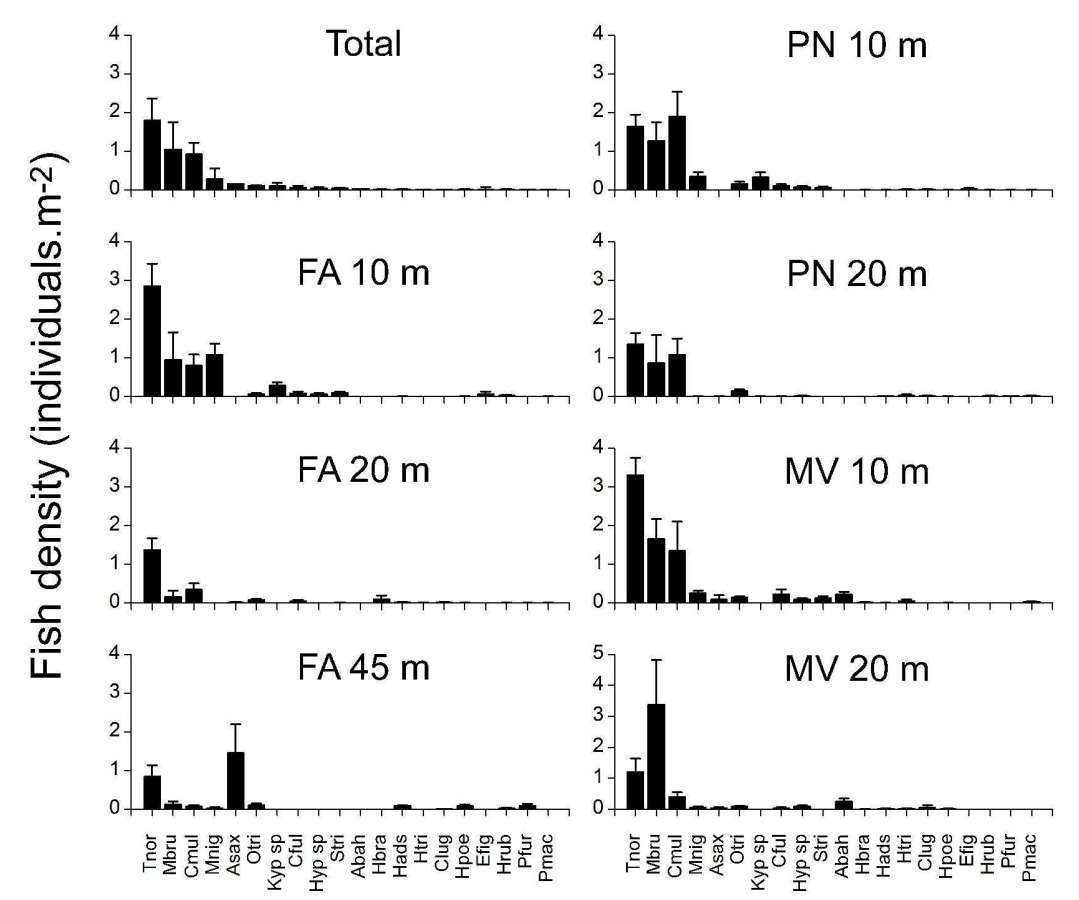

Fig. 2. Density (mean $+\mathrm{SE}$ ) of the 20 most abundant fish species FA - Enseada dos Farilhões, PN - Ponta do Noroeste and MV - Martin Vaz. Numbers denote depth strata. Note differences in scale of y axes. Abbreviations of species names: genus indicated by first capital letter and specific epithet by following letters (except for species identified to genus level only, for which the first three letters of genus name were used; see full names in Table 1).

Table 2. Benthic reef organisms recorded at Trindade (TR) and Martin Vaz (MV) Islands. Plus sign denotes presence.

\begin{tabular}{|c|c|c|c|c|c|}
\hline Taxa & TR & MV & Taxa & $\mathrm{TR}$ & MV \\
\hline Cyanobacteria & & & Rhodophyta (cont.) & & \\
\hline Lyngbya sp. & + & + & Ceramium spp. & + & + \\
\hline Chlorophyta & & & Champia parvula $^{\mathrm{a}}$ & + & + \\
\hline Anadyomene saldanhae & + & + & Dasya brasiliensis ${ }^{\mathrm{a}}$ & + & \\
\hline Anadyomene stellata & + & & Heterosiphonia crassipes $^{\text {a }}$ & + & \\
\hline Bryopsis pennata & + & + & Herposiphonia secunda f. tenella & + & \\
\hline Caulerpa kempfii & & + & Hydrolithon onkodes ${ }^{\mathrm{a}}$ & + & \\
\hline Caulerpa mexicana & + & & Lithophyllum prototypum $^{\mathrm{c}}$ & + & + \\
\hline Caulerpa pusilla & + & + & Phymatolithon masonianum ${ }^{\mathrm{a}}$ & + & \\
\hline Caulerpa verticillata & + & + & Peyssonelia sp. & + & \\
\hline Dictyosphaeria sp. & + & & Polysiphonia spp. & + & \\
\hline Halimeda discoidea & + & & Jania adhaerens & + & + \\
\hline Neomeris sp. & + & & Spongites sp. ${ }^{\mathrm{a}}$ & + & \\
\hline Rhipilia tomentosa & + & & Octocorals & & \\
\hline Udotea flabellum & + & & Plexaurela regia & + & \\
\hline Ochrophyta & & & Scleractinians & & \\
\hline Canistrocarpus cervicornis & + & + & Agaricia cf. fragilis $^{\mathrm{b}}$ & + & \\
\hline Dictyopteris delicatula & + & & Favia gravida & + & + \\
\hline Dictyopteris plagiogramma & + & & Favia leptophyla & + & + \\
\hline Dictyota ciliolata $^{\mathrm{a}}$ & + & + & Montastrea cavernosa $^{\mathrm{b}}$ & + & \\
\hline Dictyota mertensii & + & + & Mussismilia hispida & + & + \\
\hline Dictyota pulchella ${ }^{\text {a }}$ & + & & Scolymia wellsi ${ }^{\mathrm{b}}$ & + & \\
\hline Feldmannia indica ${ }^{\mathrm{a}}$ & + & & Siderastrea sp. & + & \\
\hline Lobophora variegata & + & + & Black corals (Antipatharia) & & \\
\hline Padina gymnospora & + & & Cirripathes sp. ${ }^{\mathrm{b}}$ & + & \\
\hline Rhodophyta & & & Sponges & + & + \\
\hline Amphiroa beauvoisii ${ }^{\text {a }}$ & + & & Green Sponge & + & + \\
\hline Asparagopsis taxiformis & + & & Brown Sponge & + & + \\
\hline Botryocladia pyriformis & + & + & Yellow Sponge & + & + \\
\hline Centroceras clavulatum & + & & Orange Sponge & + & + \\
\hline
\end{tabular}

New record for the TMVIG; ${ }^{b}$ Species only recorded or particularly abundant on deep reefs $(>60 \mathrm{~m}) ;{ }^{c} \mathrm{New}$ record for the South Atlantic Ocean. 


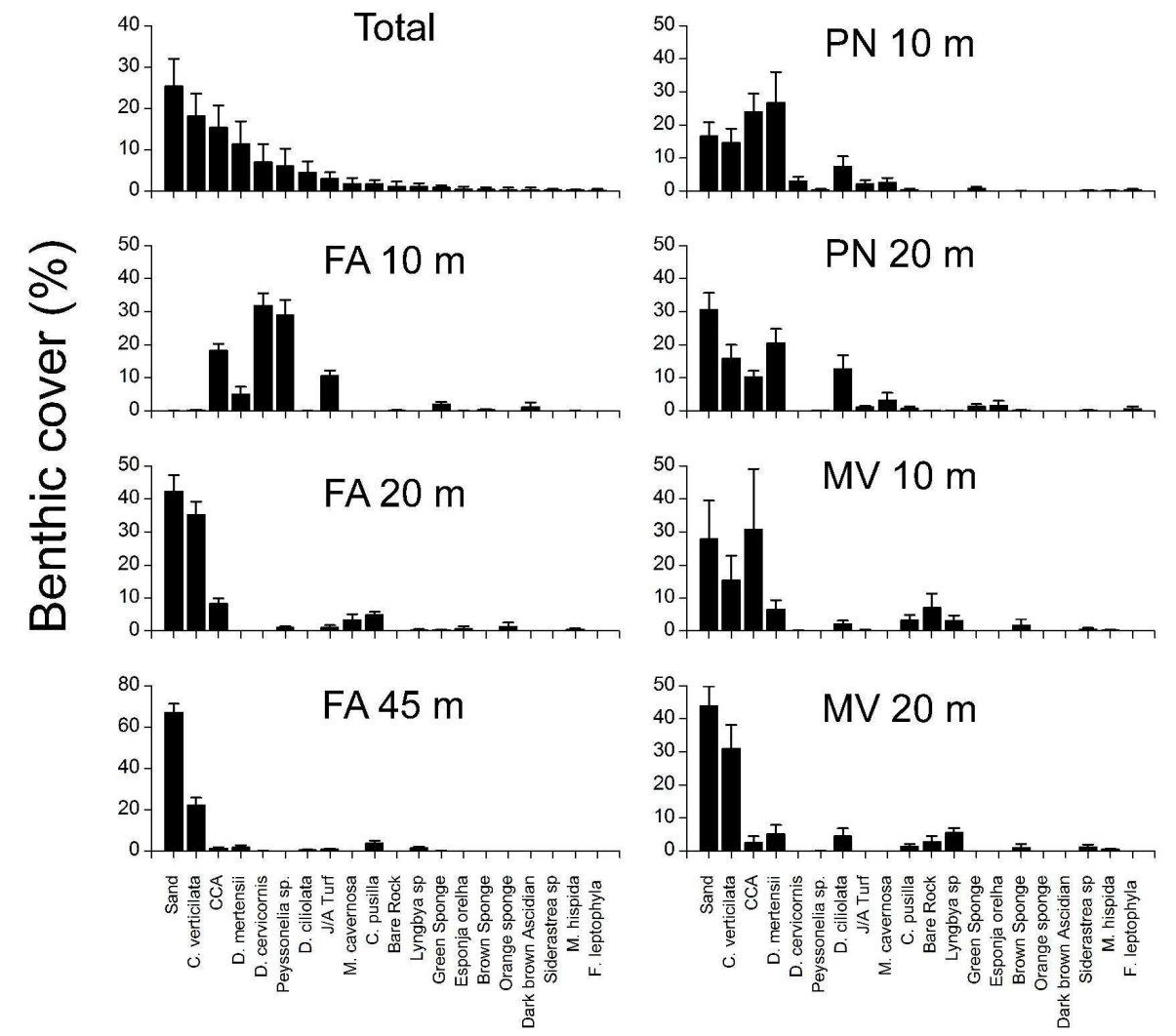

Fig. 3. Benthic cover (mean $+\mathrm{SE}$ ) of the 20 most abundant organisms (relative cover > 1\%). FA Enseada dos Farilhões, PN -Ponta do Noroeste and MV - Martin Vaz. Numbers denote depth strata. Note differences in scale of y axes.

Table 3. Two-way analysis of variance (ANOVA) testing differences in fish density among sites and depths and Tukey post-hoc comparisons. Df - degrees of freedom. Depth strata: S - shallow (10 m) and I - intermediate (20 m). Sampling sites: FA Enseada dos Farilhões, PN - Ponta do Noroeste and MV - Martin Vaz. Ns - not significant.

\begin{tabular}{|c|c|c|c|c|c|c|c|c|}
\hline \multirow[t]{2}{*}{ Fish species } & \multicolumn{2}{|c|}{$\begin{array}{c}\text { Site } \\
(\mathbf{d f}=2)\end{array}$} & \multicolumn{2}{|c|}{$\begin{array}{c}\text { Depth } \\
(\text { df }=1)\end{array}$} & \multicolumn{2}{|c|}{$\begin{array}{l}\text { Site } x \text { depth } \\
\quad(\mathbf{d f}=2)\end{array}$} & \multicolumn{2}{|c|}{ Tukey post-hoc } \\
\hline & $\mathbf{F}$ & $\mathbf{p}$ & $\mathbf{F}$ & $\mathbf{p}$ & $\mathbf{F}$ & $\mathbf{p}$ & Site & Depth \\
\hline Abudefduf saxatilis & 3.37 & $*$ & 14.88 & $* * *$ & 3.03 & ns & $\mathrm{PN}=\mathrm{FA}>\mathrm{FA}=\mathrm{MV}$ & $S>I$ \\
\hline Acanthurus bahianus & 3.47 & $*$ & 1.69 & ns & 5.70 & $* *$ & $\mathrm{FA}=\mathrm{PN}>\mathrm{PN}=\mathrm{MV}$ & $\mathrm{ns}$ \\
\hline Caranx lugubris & 0.86 & $\mathrm{~ns}$ & 0.44 & $\mathrm{~ns}$ & 1.37 & ns & $\mathrm{ns}$ & $\mathrm{ns}$ \\
\hline Cephalopholis fulva & 3.52 & $*$ & 0.87 & ns & 0.30 & ns & $\mathrm{PN}=\mathrm{MV}>\mathrm{MV}=\mathrm{FA}$ & ns \\
\hline Chromis multilineata & 5.57 & $* *$ & 0.23 & $\mathrm{~ns}$ & 1.14 & ns & $\mathrm{MV}=\mathrm{PN}>\mathrm{PN}=\mathrm{FA}$ & $\mathrm{ns}$ \\
\hline Elacatinus pridisi & 1.70 & ns & 0.02 & ns & 1.34 & ns & ns & ns \\
\hline Halichoeres brasiliensis & 1.43 & $\mathrm{~ns}$ & 0.58 & $\mathrm{~ns}$ & 7.73 & $* *$ & ns & $\mathrm{ns}$ \\
\hline Halichoeres rubrovirens & 40.58 & $* * *$ & 0.12 & ns & 0.09 & ns & $\mathrm{MV}>\mathrm{PN}=\mathrm{FA}$ & ns \\
\hline Halichoeres poeyi & 0.64 & $\mathrm{~ns}$ & 3.36 & $\mathrm{~ns}$ & 1.18 & ns & $\mathrm{ns}$ & $\mathrm{ns}$ \\
\hline Holacanthus tricolor & 0.02 & ns & 1.71 & ns & 0.06 & ns & ns & ns \\
\hline Holocentrus adscensionis & 0.04 & ns & 1.66 & ns & 0.18 & ns & ns & $\mathrm{ns}$ \\
\hline Hypleurochillus sp. & 0.36 & $\mathrm{~ns}$ & 1.20 & ns & 0.36 & ns & ns & $\mathrm{ns}$ \\
\hline Kyphosus sectator & 1.03 & $\mathrm{~ns}$ & 9.61 & $* *$ & 1.26 & ns & ns & $S>I$ \\
\hline Malacoctenus brunoi & 5.46 & $* *$ & 40.04 & $* * *$ & 6.73 & $* *$ & $\mathrm{FA}>\mathrm{MV}=\mathrm{PN}$ & $S>I$ \\
\hline Melichthys Níger & 2.74 & $\mathrm{~ns}$ & 3.76 & ns & 0.05 & ns & ns & ns \\
\hline Ophioblennius trinitatis & 1.35 & ns & 31.83 & $* * *$ & 1.10 & ns & ns & $S>I$ \\
\hline Cephalopholis furcifer & 2.59 & ns & 0.05 & $\mathrm{~ns}$ & 0.65 & ns & ns & ns \\
\hline Pseudupneneus maculatus & 2.08 & ns & 2.19 & ns & 0.54 & ns & ns & ns \\
\hline Stegastes trindadensis & 3.50 & $*$ & 4.93 & $*$ & 0.83 & ns & $\mathrm{MV}=\mathrm{PN}>\mathrm{PN}=\mathrm{FA}$ & $S>I$ \\
\hline Thalassoma noronhanum & 1.18 & ns & 12.22 & $* *$ & 2.05 & ns & ns & $S>I$ \\
\hline Total fish density & 3.43 & $* * *$ & 15.21 & $* * *$ & 1.64 & ns & $\mathrm{MV}=\mathrm{PN}>\mathrm{PN}=\mathrm{FA}$ & $S>I$ \\
\hline Large carnivores & 0.79 & ns & 0.73 & ns & 1.72 & ns & ns & ns \\
\hline
\end{tabular}

$* \mathrm{P}<0.05, * * \mathrm{P}<0.01, * * * \mathrm{P}<0.001$ and ns - not significant. 
Table 4. Two-way analysis of variance (ANOVA) testing differences in benthic cover among sites and depths and Tukey posthoc comparisons. Df - degrees of freedom. Depth strata: S - shallow (10 m) and I - intermediate (20 m). Sampling sites: FA Enseada dos Farilhões, PN - Ponta do Noroeste and MV - Martin Vaz. Ns - not significant.

\begin{tabular}{|c|c|c|c|c|c|c|c|c|}
\hline \multirow[t]{2}{*}{ Benthic category / species } & \multicolumn{2}{|c|}{$\begin{array}{c}\text { Site } \\
(\mathbf{d f}=2)\end{array}$} & \multicolumn{2}{|c|}{$\begin{array}{c}\text { Depth } \\
(\mathrm{df}=1)\end{array}$} & \multicolumn{2}{|c|}{$\begin{array}{l}\text { Site } x \text { depth } \\
\quad(\mathrm{df}=2)\end{array}$} & \multicolumn{2}{|c|}{ Tukey post-hoc } \\
\hline & $\mathbf{F}$ & $\mathbf{p}$ & $\mathbf{F}$ & $\mathbf{p}$ & $\mathbf{F}$ & $\mathbf{p}$ & Site & Depth \\
\hline \multicolumn{9}{|l|}{ Ascidian } \\
\hline Dark-brown Ascidian & 0.83 & ns & 0.71 & ns & 0.83 & ns & ns & ns \\
\hline \multicolumn{9}{|l|}{ Coral } \\
\hline Montastrea cavernosa & 2.51 & ns & 1.33 & ns & 0.78 & ns & $\mathrm{ns}$ & ns \\
\hline Mussismilia híspida & 4.49 & $*$ & 1.74 & ns & 4.89 & $*$ & $\mathrm{MV}>\mathrm{FA}>\mathrm{PN}$ & $\mathrm{ns}$ \\
\hline Siderastrea $\mathrm{sp}$. & 7.19 & $* * *$ & 0.92 & $\mathrm{~ns}$ & 0.61 & ns & $\mathrm{MV}>\mathrm{PN}>\mathrm{FA}$ & ns \\
\hline Favia leptophyla & 1.59 & ns & 0.08 & ns & 0.09 & ns & ns & $\mathrm{ns}$ \\
\hline Total & 1.23 & ns & 2.11 & ns & 0.66 & $\mathrm{~ns}$ & ns & ns \\
\hline \multicolumn{9}{|l|}{ Crustose Coralline Algae } \\
\hline Peyssonelia sp. & 82.77 & $* * *$ & 41.94 & $* * *$ & 46.24 & $* * *$ & $\mathrm{FA}>\mathrm{PN}>\mathrm{MV}$ & $S>I$ \\
\hline Other & 0.94 & ns & 15.21 & $* * *$ & 1 & $\mathrm{~ns}$ & ns & $\mathrm{S}>\mathrm{I}$ \\
\hline Total & 2.71 & ns & 29.36 & $* * *$ & 2.5 & $\mathrm{~ns}$ & $\mathrm{~ns}$ & $\mathrm{~S}>\mathrm{I}$ \\
\hline \multicolumn{9}{|l|}{ Fleshy macroalgae } \\
\hline Canistrocarpus cervicornis & 43.64 & $* * *$ & 77.53 & $* * *$ & 43.68 & $* * *$ & $\mathrm{FA}>\mathrm{PN}>\mathrm{MV}$ & $S>I$ \\
\hline Dictyota ciliolata & 18.57 & $* * *$ & 1.84 & ns & 0.72 & $\mathrm{~ns}$ & $\mathrm{PN}>\mathrm{MV}>\mathrm{FA}$ & $\mathrm{ns}$ \\
\hline Dictyota mertensii & 14.95 & $* * *$ & 1.41 & ns & 1.17 & ns & $\mathrm{PN}>\mathrm{MV}>\mathrm{FA}$ & $\mathrm{ns}$ \\
\hline Total & 7.59 & $* *$ & 5.92 & $*$ & 5.25 & $* *$ & $\mathrm{PN}>\mathrm{FA}=\mathrm{MV}$ & $S>I$ \\
\hline \multicolumn{9}{|l|}{ Sponge } \\
\hline Green Sponge & 8.26 & $* * *$ & 1.77 & ns & 6.22 & $* *$ & $\mathrm{FA}=\mathrm{PN}>\mathrm{MV}$ & $\mathrm{ns}$ \\
\hline Brown Sponge & 1.94 & ns & 0.38 & ns & 0.34 & ns & ns & $\mathrm{ns}$ \\
\hline Yellow Sponge & 0.73 & ns & 1.89 & ns & 0.84 & $\mathrm{~ns}$ & $\mathrm{~ns}$ & $\mathrm{~ns}$ \\
\hline Orange Sponge & 1.11 & ns & 0.95 & ns & 1.11 & $\mathrm{~ns}$ & ns & $\mathrm{ns}$ \\
\hline Total & 0.31 & ns & 0.24 & ns & 1.1 & ns & $\mathrm{ns}$ & ns \\
\hline \multicolumn{9}{|l|}{ Stoloniferous Algae } \\
\hline Caulerpa verticillata & 2.28 & ns & 37.57 & $* * *$ & 14.71 & $* * *$ & $\mathrm{~ns}$ & $\mathrm{I}>\mathrm{S}$ \\
\hline Caulerpapusilla & 5.02 & $* *$ & 10.33 & $* * *$ & 14.97 & $* * *$ & $\mathrm{MV}>\mathrm{FA}>\mathrm{PN}$ & $\mathrm{I}>\mathrm{S}$ \\
\hline Total & 2.16 & ns & 26.24 & $* * *$ & 11.97 & $* * *$ & ns & $\mathrm{I}>\mathrm{S}$ \\
\hline \multicolumn{9}{|l|}{ Turf } \\
\hline Jania plus Amphiroa & 28.21 & $* * *$ & 24.25 & $* * *$ & 14.73 & $* * *$ & $\mathrm{FA}>\mathrm{PN}>\mathrm{MV}$ & $\mathrm{S}>\mathrm{I}$ \\
\hline Lyngbya sp. & 39.45 & $* * *$ & 7.39 & $* *$ & 1.6 & $\mathrm{~ns}$ & $\mathrm{MV}>\mathrm{PN}=\mathrm{FA}$ & $\mathrm{I}>\mathrm{S}$ \\
\hline Total & 9.15 & $* * *$ & 8.52 & $* *$ & 13.66 & $* * *$ & $\mathrm{FA}>\mathrm{PN}=\mathrm{MV}$ & $S>I$ \\
\hline \multicolumn{9}{|l|}{ Abiotic } \\
\hline Bare Rock & 11.43 & $* * *$ & 2.71 & ns & 2.12 & ns & $\mathrm{MV}>\mathrm{FA}=\mathrm{PN}$ & $\mathrm{ns}$ \\
\hline Sand & 3.89 & * & 32.26 & $* * *$ & 5.43 & ** & $\mathrm{MV}>\mathrm{FA}=\mathrm{PN}$ & $\mathrm{I}>\mathrm{S}$ \\
\hline Total & 8.75 & $* * *$ & 34.79 & $* * *$ & 7.35 & $* *$ & $\mathrm{MV}>\mathrm{FA}=\mathrm{PN}$ & $\mathrm{I}>\mathrm{S}$ \\
\hline
\end{tabular}

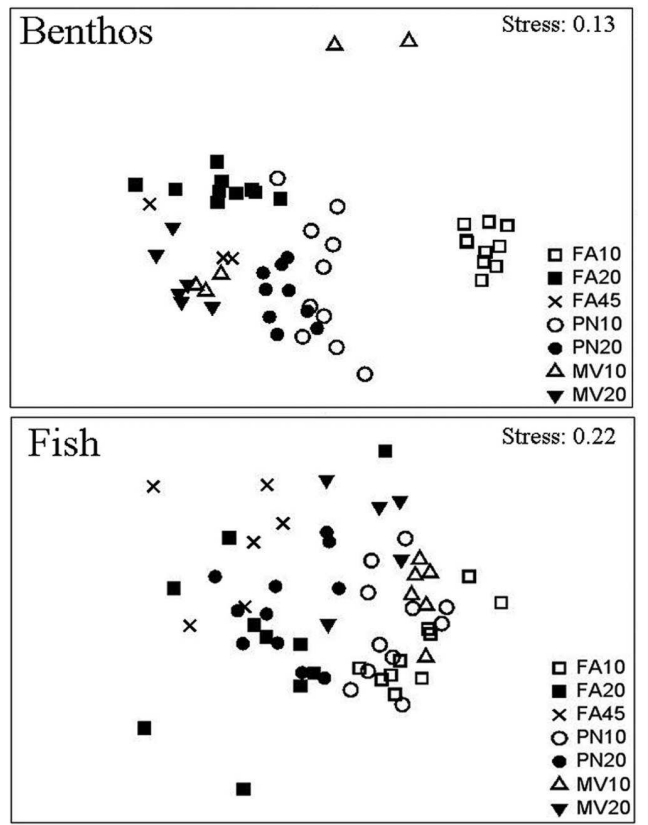

Fig. 4. Non-metric multi-dimensional scaling (nMDS) ordination based on Bray-Curtis similarities. A) Fish assemblages and B) benthic assemblages. FA - Enseada dos Farilhões, PN -Ponta do Noroeste and MV - Martin Vaz. Numbers denote depth strata. 


\section{Influence of Depth and Benthic Cover on Fish Assemblages}

Results from the CCA showed that depth was the main predictor of fish assemblage structure, followed by cover of crustose coralline algae, turf algae, ascidians, sand, fleshy macroalgae and sponges, successively. The first two axes explained $79.4 \%$ of the relationship between habitat characteristics and fish assemblage structure. Most of the fish species were associated with shallow benthic communities, characterized by crustose coralline algae, fleshy macroalgae and sponges. Four species (Cephalopholis furcifer, Holacanthus tricolor, Holocentrus adscensionis and Pseudopeneus maculatus) were associated with deep reefs (i.e., the $45 \mathrm{~m}$ depth stratum at the FA site), where stoloniferous algae and sand patches covered the rocky bottom (Fig. 5). This latter result corroborates the qualitative observations on fish assemblages of deep reefs (see above).
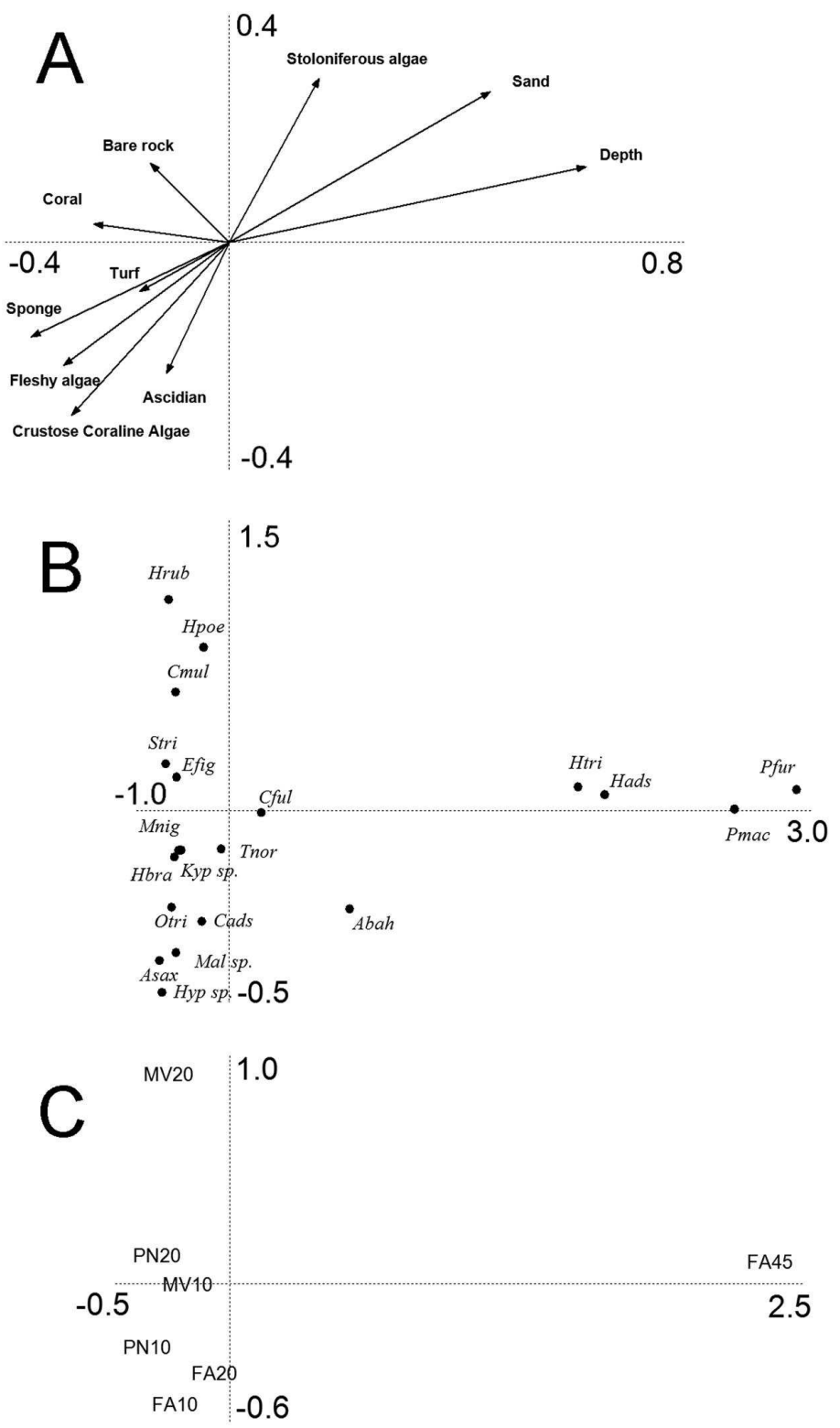

Fig. 5. Canonical correspondence analysis plot showing (A) habitat characteristics, (B) fish species and (C) sampling sites. FA Enseada dos Farilhões, PN -Ponta do Noroeste and MV - Martin Vaz. Numbers denote depth strata. Abbreviations of species names: genus indicated by first capital letter and specific epithet by following letters (except for species identified to genus level only, for which the first three letters of genus name were used; see full names in Table 1). 


\section{DiscUSSION}

Similarly to the other three Brazilian oceanic islands (i.e., Atol das Rocas, Fernando de Noronha and St. Paul's Rocks), the TMVIG possesses poor fish and benthic assemblages. In fact, the relatively small size and isolation from the mainland are the two most important and widely recognized factors leading to lower species richness among oceanic islands (SANDIN et al., 2008).

Despite the biological connectivity between the TMVIG and mainland (i.e., the eastern Brazilian coast), facilitated by the seamounts of the VitoriaTrindade Chain which work as a series of "stepping stones" (PINHEIRO et al., 2009), the structure of fish assemblages of the TMVIG is more closely similar to those of Atol das Rocas and Fernando de Noronha (ROSA; MOURA, 1997; FRANCINI-FILHO et al., 2000) than to those of the mainland. For these three oceanic islands, three fish species (Talassoma noronhanum, Chromis multilineata and Abudefduf saxatilis) dominate the assemblages, with $C$. fulva and $M$. niger also featuring as conspicuous elements. In addition, benthic assemblages of all Brazilian oceanic islands are dominated by macroalgae (fleshy and crustose), with sparse patches of other benthic organisms (especially sponges and a few coral species). Thus, other than the possibility of periodic colonization from a main but remote source (the mainland), the maintenance of these isolated populations may rely primarily on their ability to maintain viable populations in small isolated oceanic habitats. Exceptions to this pattern are the reef coral Favia leptophylla and the Brazilian wrasse Halichoeres brasiliensis. Favia leptophylla was previously considered endemic to the eastern Brazilian coast (LEÃO et al., 2003), but it is now known to occur in the TMVIG as well, possibly because of the connection promoted by the VTC. Halichoeres brasiliensis is common in the mainland (e.g. FRANCINI-FILHO; MOURA, 2008) and the TMVIG, but is absent from the other Brazilian oceanic islands, again corroborating the hypothesis of greater connectivity between mainland and the TMVIG through the VTC.

Depth, as expected, was the most important environmental variable affecting the structure of fish assemblages (see FRANCINI-FILHO; MOURA, 2008, for similar results). The influence of depth may occur via the alteration of physical variables (e.g., pressure, salinity and luminosity) or through the modification of benthic assemblages, this latter serving as shelter and/or food for fish. The shallow portion of the TMVIG is dominated by fleshy macroalgae and crustose coraline algae, a pattern possibly related to high irradiance levels promoted by the sediment-free and oligotrophic oceanic waters, and by the scarcity of specialized herbivores such as parrotfishes (family Scaridae) (FRANCINI-FILHO et al. 2010). Densities of Sparisoma amplum and Sparisoma axillare were relatively low in the TMVIG. The abundant Acanthurus bahianus, Kyphosus sectator and Melichthys niger were frequently recorded feeding upon turf and macroalgae. Despite a significant spatial variation in turf and macroalgae cover (the former dominating the shallowest strata of the FA site and the latter most abundant throughout the entire depth gradient of the PN site), no similar spatial variation in the density of the above-mentioned fishes (see Table 3) was detected. Detailed studies are still needed in order to better understand the possible role of herbivorous fishes in mediating competition between algae and other benthic organisms in the TMVIG.

Similarly to herbivores, most fish species showed little between-sites spatial variation, despite marked differences in benthic cover (see Tables 3 and 4). Exceptions to this pattern include Halichoeres rubrovirens, Chromis multilineata and Stegastes fuscus trindadensis, all of them most abundant at the same site (Martin Vaz) in which coral cover was relatively higher. The positive relationship between coral cover and reef fish abundance/species diversity is widely recognized (e.g. BELL; GALZIN, 1984), but again additional studies are needed in order to better understand the relationship between fish abundance and coral cover in the TMVIG.

The direct observations undertaken here with advanced mixed gas diving (TRIMIX) and ROV operations in the mesophotic reef ecosystems of the TMVIG are the first of their kind in Brazil. The results obtained indicate that the mesophotic reef ecosystems of the TMVIG are similar to those in other portions of the world, particularly in the Caribbean, where planktivorous fishes, black corals and platelike/massive coral species dominate (KAHNG et al., 2010). The large rhodolith beds that separate the deepest portion of the shallow rocky reef (at about 45 $m$ depth) from the deep rocky reefs at the insular shelf break (at about 80-90 m) may play an important role in the connectivity between these shallow and deep zones, mainly by providing corridors of complex habitat. In this regard, the nests of Malacanthus plumieri, which are formed by mounds of living rhodoliths, may be of particular importance.

Most colonies of the Brazilian endemic reef coral Mussismilia hispida were bleached, diseased or partially dead at the time of the sampling. The same situation was observed in the Fernando de Noronha Archipelago, off northeastern Brazil, in November 2009 (authors' pers. obs.). The causes of the decline in the vitality of $M$. hispida on these two Brazilian oceanic islands are still unknown, but an increase in sea surface temperature and infection by pathogenic 
bacteria cannot be disregarded, as both factors have negatively affected the health of corals (including $M$. hispida) in eastern Brazil (FRANCINI-FILHO et al., 2008; ALVES-JR et al., 2010).

Signs of overfishing in the TMVIG are suggested by the relatively low density of large carnivorous fishes $(0.03 \pm 0.01$ individuals.m-2) such as Epinephelus adscensionis and Sphyraena barracuda. Comparative values for this same group in the fully-protected area of the Abrolhos Archipelago, Eastern Brazil, are $0.05 \pm 0.02$ individuals.m-2 (authors' unpub. data). Additional weight is lent to this hypothesis by the sighting of only two sharks during the entire expedition (a Ginglymostoma cirratum individual in Trindade and a Charcharhinus perezi in Martin Vaz). Recent studies show that the TMVIG is an appreciated fishing ground, with Caranx lugubris, Cephalopholis fulva and Epinephelus adscensionis figuring among the most important target species for the hook and line fishery (PINHEIRO; GASPARINI, in press). Besides evidence of overfishing, the TMVIG possess five endemic and restricted range fish species (Elacatinus pridisi, Halichoeres rubrovirens, Scartella poiti, Sparisoma rocha and Stegastes fuscus trindadensis), as well as at least three new, but still undescribed reef fish species (PINHEIRO et al., 2009; C.A. RANGEL, pers. comm.). Considering the evidence of overfishing and the fragility and uniqueness of the ecosystems of isolated oceanic islands (ALLEN, 2008), there is a clear need to implement precautionary conservation measures, most importantly the establishment of a permanent no-take zone encompassing the entire TMVIG.

\section{ACKNOWLEDGEMENTS}

We wish to thank Linda G. Waters for reviewing the manuscript. Wladimir C. Paradas and the crew of Cat. Guruçá for field assistance. Financial support was provided by the Brazilian Research Council (CNPq; grants to S.M.P.B. Guimarães and G.M. Amado Filho). G.H. Pereira Filho recognises his debt to CAPES for a post doctoral scholarship. Essential logistical support was provided by Conservation International Brazil.

\section{REFERENCES}

ALLEN, G. R. Conservation hotsposts of biodiversity and endemism for Indo-Pacific coral reef fishes. Aquatic Conserv. mar. freshwater Ecosys., v. 18, n. 5, p.541556, 2008.

ALVES, R. J. V.; CASTRO, J. W. A. (Ed.). Ilhas oceânicas brasileiras: da pesquisa ao manejo. Brasília, DF: IBAMA, Ministério do Meio Ambiente, 2006. 299 p.

ALVES-JR, N.; NETO, O. S. M.; SILVA, B. S. O.; MOURA, R. L.; FRANCINI-FILHO, R. B.; CASTRO, C. B.; PARANHOS, R.; BITNER-MATHÉ, B. C.;
KRUGER, R. H.; VICENTE, A. C. P.; THOMPSON, C. C.; THOMPSON, F. L. Diversity and pathogenic potential of vibrios isolated from Abrolhos Bank corals. Environ. Microbiol. Repts, v. 2, p. 90-95, 2010.

BELL, J. D.; GALZIN, R. Influence of live coral cover on coral reef fish communities. Mar. Ecol. Prog. Ser., v 15, p. 265-274, 1984.

CLARKE, K. R.; WARWICK, R. M. Change in marine communities: An approach to statistical analysis and interpretation. Plymouth: Plymouth Marine Laboratory, 1994. $144 \mathrm{p}$.

FLOETER, S. R.; ROCHA, L. A.; ROBERTSON, D.R.; JOYEUX, J. C.; SMITH-VANIZ, W. F.; WIRTZ, P.; EDWARDS, A. J.; BARREIROS, J. P.; FERREIRA, C. E. L.; GASPARINI, J. L.; BRITO, A.; FALCÓN, J.M.; BOWEN, B.W.; BERNARDI, G. Atlantic reef fish biogeography and evolution. J. Biogeogr., v. 35, p. 22$47,2008$.

FRANCINI-FILHO, R. B.; MOURA R. L. Dynamics of fish assemblages on coral reefs subjected to different management regimes in the Abrolhos Bank, eastern Brazil. Aquatic Conserv. mar. freshwater Ecosys., v. 18 , p. $1166-1179,2008$.

FRANCINI-FILHO, R. B; MOURA R. L.; SAZIMA, I. Cleaning by the wrasse Thalassoma noronhanum, with two records of predation by its grouper client Cephalopholis fulva. J. Fish Biol, v. 56, p. 802-809, 2000.

FRANCINI-FILHO, R. B.; MOURA, R. L.; THOMPSON, F. L.; REIS, R. D.; KAUFMAN, L.; KIKUCHI, R. K. P.; LEÃO, Z. M. A. N. Diseases leading to accelerated decline of reef corals in the largest South Atlantic reef complex (Abrolhos Bank, eastern Brazil). Mar. Pollut. Bull., v. 56, n. 5, p. 1008 - 1014, 2008.

FRANCINI-FILHO, R. B.; FERREIRA, C. M.; CONI, E. C. O.; MOURA, R. L.; KAUFMAN, L. Foraging activity of roving herbivorous reef fish (Acanthuridae and Scaridae) in eastern Brazil: influence of resource availability and interference competition. J. mar. Biol. Ass. U.K. (Print), v. 90, p. 481-492, 2010.

GASPARINI, J. L.; FLOETER, S. R. The shore fishes of Trindade Island, Western South Atlantic. J. nat. Hist., v. 35, p. 1639-1656, 2001.

KAHNG, S. E.; GARCIA-SAIS, J. R.; SPALDING, H. L.; BROKOVICH, E.; WAGNER, D.; WEIL, E.; HINDERSTEIN, L.; TOONEN, R. J. Community ecology of mesophotic coral reef ecosystems. Coral Reefs, v. 29, n. 2, p. 255-275, 2010.

KIER, G.; KREFT, H.; LEE, T. M.; JETZ, W.; IBISCH, P. L; NOWICKI, C.; MUTKE, J.; BARTHLOTT, W. A Global assessment of endemism and species richness across island and mainland regions. Proc. natn Acad. Sci., v. 106, n. 23, p. 9322-9327, 2009.

KOHLER, K. E.; GILL, S. M. Coral Point Count with Excel extensions (CPCe): A Visual Basic program for the determination of coral and substrate coverage using random point count methodology. Computers, Geosciences, v. 32, n. 9, p. 1259-1269, 2006.

LAVRADO, H. P. Caracterização do ambiente e da comunidade bentônica. In: LAVRADO, H.P.; IGNACIO, B. L. (Ed.). Biodiversidade bentônica da região central da Zona Econômica Exclusiva Brasileira. Rio de Janeiro: Museu Nacional, 2006. p. 1964. (Série Livros, n. 18). 
LEÃO, Z. M. A. N.; KIKUCHI, R. K. P.; TESTA, V. Corals and coral reefs of Brazil. In: CORTÉS, J. (Ed.). Latin American coral reefs. Amsterdam: Elsevier Science, 2003. p. 9-52.

MARQUES, L. S.; ULBRICH, M. N. C.; RUBERTI, E.; TASSINARI, C. G. Petrology, geochemistry and Sr-Nd isotopes of the Trindade and Martin Vaz volcanic rocks (Southern Atlantic Ocean). J. Volcan. Geotherm. Res., v. 93, p.191-216, 1999.

MINTE-VERA, C. V.; MOURA R .L.; FRANCINI-FILHO R. B. Nested sampling: An improved visual-census technique for studying reef fish assemblages. Mar. Ecol. Prog. Ser., v. 367, p. 283-293, 2008.

MORAES, F.; VENTURA, M.; KLAUTAU, M.; HAJDU, E.; MURICY, G. Biodiversidade de esponjas das ilhas oceânicas brasileiras. In: ALVES, R.J.V.; CASTRO, J.W.A. (Ed.). Ilhas oceânicas brasileiras: da pesquisa ao manejo. Brasília, DF: IBAMA, Ministério do Meio Ambiente, 2006. p. 148-178.

NASSAR, C. A. G. An assessment to the benthic marine algae at Trindade Island, Espírito Santo, Brazil. Rev. Bras. Biol., v. 54, p. 623-629, 1994.

PEDRINI, A. G.; GONÇALVES, J. E. A; FONSECA, M. C. S.; ZAÚ, A. S.; LACORTE, C. C. A survey of the marine algae of Trindade Island, Brazil. Bot. Mar., v. 32, p. 97-99, 1989.

PINHEIRO, H. T.; GASPARINI, J. L. Peixes recifais do complexo insular oceânico Trindade-Martin Vaz: novas ocorrências, atividades de pesca e mortandade natural. Brasília,DF: Ministério do Meio Ambiente. (In press).

PINHEIRO, H. T.; CAMILATO, V.; GASPARINI, J. L.; JOYEUX, J. New records of fishes for Trindade-Martin Vaz oceanic insular complex, Brazil. Zootaxa, v. 2298, p. 45-54, 2009.

ROSA, R. S.; MOURA, R. L. Visual assessment of reef fish community structure in the Atol das Rocas Biological Reserve, off north-eastern Brazil. In: INTERNATIONAL CORAL REEF SYMPOSIUM, 8., 1997, Panama. Proceedings...n. 1, p. 983-986, 1997.

RUSS, G. R.; ALCALA, A. C. Natural fishing experiments in marine reserves 1983-1993: roles of life history and fishing intensity in family responses. Coral Reefs, v. 17, p. 399-416, 1998.
SANDIN, S. A.; VERMEIJ, M. J. A.; HURLBERT, A.H. Island biogeography of Caribbean coral reef fish. Global Ecol. Biogeogr., v. 17, p. 770-777, 2008.

SILVEIRA, I. C.; SCHMIDT, A. C. K.; CAMPOS, E. J. D.; GODOI, S. S.; IKEDA, I. A. Corrente do Brasil ao largo da costa leste brasileira. Rev. Bras. Oceanogr., v. 48, n. 2, p. 171-183, 2000.

STENECK, R. S.; DETHIER, M. N. A functional group approach to the structure of algal-dominated communities. Oikos, v. 69, p. 476-498, 2004.

TER BRAAK, C. J. F. Unimodal models to relate species to Environment. Wageningen: DLO-Agricultural Mathematics Group, 1996.

VILLAÇA, R.; PEDRINI, A. G.; PEREIRA, S. M. B.; FIGUEIREIDO, M. A. O. Flora marinha bentônica das ilhas oceânicas brasileiras. In: ALVES, R. J. V.; CASTRO, J. W. A. (Ed.). Ilhas oceânicas brasileiras: da pesquisa ao manejo. Brasília, DF: IBAMA, Ministério do Meio Ambiente, 2006. p. 107-146.

WHITTAKER, R. J.; FERNANDEZ-PALACIOS, J. M. Island biogeography: ecology, evolution, and conservation. Oxford: Oxford Univ. Press., 2007

YONESHIGUE-VALENTIN, Y.; FERNANDES, D. R. P.; PEREIRA, C. B.; RIBEIRO, S. M. Macroalgas da plataforma continental da Ilha da Trindade e do Arquipélago de Martin Vaz (Espírito Santo, Brasil). In: REUNIÃO BRASILEIRA DE FICOLOGIA, 10, Rio de Janeiro. Anais... Rio de Janeiro, Museu Nacional, 2005, p. 361-372. (Série Livros n. 10).

YONESHIGUE-VALENTIN, Y.; GESTINARI, L. M. S.; FERNANDES, D. R. P. Macroalgas. In: LAVRADO, $\mathrm{H}$. P.; IGNACIO, B. L. (Ed.). Biodiversidade bentônica da região central da Zona Econômica Exclusiva Brasileira. Rio de Janeiro: Museu Nacional, 2006. p. 67-105. (Série Livros n. 18).

ZAR, J. H. Biostatistical analysis. Upper Saddle River, NJ: Prentice-Hall, 1999. 